\title{
Changes in Bone Mineral Density Following Conventional Oral Phosphonate Treatment of Hypophosphatemic Osteomalacia: A Non-Randomized Controlled Study
}

Yue Guo, ${ }^{1,2, *}$ Ying-Hui Zhou, ${ }^{1, *}$ Xian-Ping Wu,' Chen-

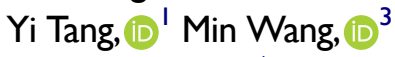
Zhao-Hui Mo, (D) ${ }^{4}$ John A Shepherd, (D) ${ }^{5}$ Bennett K Ng, ${ }^{5}$ Bo Fan, ${ }^{5}$ Hou-De Zhou'

'National Clinical Research Center for Metabolic Diseases, Hunan Provincial Key Laboratory of Metabolic Bone Diseases, and Department of Metabolism and Endocrinology, The Second Xiangya Hospital of Central South University, Changsha, 4I00II, Hunan, People's Republic of China; ${ }^{2}$ Department of Stomatology, The Second Xiangya Hospital, Central South University, Changsha, 4100II, Hunan, People's Republic of China; ${ }^{3}$ Department of Endocrinology, Xiangya Hospital of Central South University, Changsha, 410008, People's Republic of China; ${ }^{4}$ Department of Endocrinology, The Third Xiangya Hospital of Central South University, Changsha, 4I00 13, People's Republic of China; ${ }^{5}$ Department of Radiology \& Biomedical Imaging, University of California at San Francisco, San Francisco, CA, 94I43, USA

*These authors contributed equally to this work

Correspondence: Hou-De Zhou National Clinical Research Center for Metabolic Diseases, Hunan Provincial Key Laboratory for Metabolic Bone Diseases, and Department of Metabolism and Endocrinology, The Second Xiangya Hospital of Central South University, Changsha, 4I00II, Hunan, People's Republic of China

Tel +86-73I-85292223

Fax +86-73I-85533525

Email houdezhou@csu.edu.cn
Purpose: There are limited clinical studies aimed at solving the problem of the efficiency of conventional treatment with oral phosphate and calcitriol in adults with hypophosphatemic osteomalacia (HO). In addition, there still had no good non-hazardous markers to evaluate the severity of bone loss of osteomalacia before and after treatment. Therefore, the purpose of this study was to assess the efficacy of conventional treatment with a self-blended phosphate supplementation and calcitriol on patients with $\mathrm{HO}$ and whether bone mineral density (BMD) can be helpful for monitoring the efficacy.

Patients and Methods: A total of $21 \mathrm{HO}$ patients and 105 healthy controls were enrolled. All patients were tested for serum biomarkers and BMD of the lumbar spine (L1-L4), femoral neck, and total left hip. After three years of treatment, 11 of $21 \mathrm{HO}$ patients were recalled for BMD measurement. According to the administration of drugs, $\mathrm{HO}$ patients with calcium and calcitriol were divided into three phosphate treatment groups: patients in group $\mathrm{A}(\mathrm{n}=3)$ received continuous phosphate supplementation, patients in group $B(n=5)$ received intermittent phosphate supplementation and patients in group $\mathrm{C}(\mathrm{n}=3)$ received no phosphate supplementation.

Results: The diagnoses of $21 \mathrm{HO}$ patients were 5 cases of hereditary hypophosphatemic rickets, 4 cases of Fanconi syndrome with the features of renal tubular acidosis and vitamin D deficiency, and 12 cases of hereditary vitamin D abnormality. The average initial serum phosphorus level of the patient group was approximately 50\% lower than that of the control group. Lower BMD was significantly observed in the HO group than the control group at the lumbar spine and total hip. Continuous treatment with the phosphate supplement could increase BMD in the lumbar spine and total hip by $33.4-52.3 \%$ and in the femoral neck increased by $43.2-79.3 \%$ compared with baseline, and the effect appears to be continued once treatment is discontinued.

Conclusion: These findings suggest that conventional therapy can improve bone mineral defects in patients with HO, especially in the femoral neck. Detection of BMD in HO patients is a good tool to assess the extent of bone defects and the therapeutic effect.

Trial Registration: Chinese Clinical Trial Registry, ChiCTR-OOC-16010095. Registered 7 December 2016. Retrospectively registered.

Keywords: bone mineral density, conventional treatment, hypophosphatemic osteomalacia

\section{Introduction}

Phosphorus, which is ranked as the second most enriched mineral in human body, plays a very important role in a variety of biological processes. Chronic hypophosphatemia usually occurs due to increased renal phosphate loss (such as specific hereditary genetic 
mutations - X-linked hypophosphatemia, Fanconi syndrome and renal tubular acidosis), decreased intestinal phosphate absorption (such as malnourishment and short bowel syndrome), and extracellular phosphorus migration to cells or bone (such as vitamin D deficiency). ${ }^{1}$ This condition decreases serum phosphorus (P) and calcium (Ca) concentrations, 2,3 and usually impairs mineralization of the bone matrix and results in abnormal growth and rickets in children, and osteomalacia in adults. It is recommended to use traditional medical treatment with oral phosphate and calcitriol for children. However, it is uncertain whether conventional medical treatment is continuously effective for adults and the efficacy has not yet been proved. ${ }^{4}$ There are limited clinical studies that showed conventional therapy is effective for adults. Sullivan et al conducted open-label research which showed that the traditional medical treatment can reduce the severity of osteomalacia in symptomatic adults from the quantified data of pre- and post-treatment bone biopsies. $^{5}$

The main purposes of therapy for hypophosphatemic osteomalacia (HO) in adults are to relieve pain and the severity of osteomalacia and/or to promote fracture healing or surgical recovery. ${ }^{6}$ Bone biopsies and serum levels of alkaline phosphatase, $\mathrm{P}, \mathrm{Ca}$ are often used to assess the therapeutic response of osteomalacia. However, it is too invasive to use bone biopsies routinely and it is also not enough to use biochemical parameters to evaluate the endpoints of treatment due to the time course and eventual extent of recovery in bone mineral defects are various at different skeletal sites. ${ }^{7}$ There still has no good non-hazardous markers to evaluate the extent of the osteomalacia before and after therapy. Although bone mineral density (BMD) detected by dual-energy X-ray absorptiometry (DXA) is not necessary for the diagnosis of osteomalacia, many studies showed that osteomalacia may lead to decreased $\mathrm{BMD},{ }^{7-13}$ which in turn leads to increased risk of fracture. In addition, it has been illustrated that the BMD of the spine and femur is significantly correlated with quantitative histological measurement in patients with a variety of metabolic bone diseases including osteomalacia. $^{14}$ Thus, the measurement of BMD in patients with $\mathrm{HO}$ is important and maybe also beneficial for evaluation of the extent of bone defects and the following management.

In the present study, we, therefore, performed a selfblended oral phosphate supplement and calcitriol on patients with $\mathrm{HO}$ and measured the BMD of pre- and post-treatment to assess the efficacy of conventional therapy and whether BMD can be helpful for monitoring the efficacy.

\section{Materials and Methods Participants}

In this retrospective non-randomized controlled study, 21 HO participants (13 males and 8 females) were identified as the case group through medical record review at three affiliated hospitals of Central South University (Xiangya Hospital, the Second Xiangya Hospital, the third Xiangya Hospital) from January 1, 2006 to September 1, 2013. One hundred and five age, sex, height, and weight matched participants with normal blood biochemical and BMD measurements and no systemic disease were collected as the control group. This study was conducted in accordance with the Declaration of Helsinki and was approved by the Ethics Committee of The Second Xiangya Hospital of Central South University (No. 2012-226). Informed consent was obtained from the subjects before all measurements. Our study adheres to CONSORT guidelines of clinical trials.

The diagnoses of $21 \mathrm{HO}$ patients were as follows: hereditary hypophosphatemic rickets by genetic testing $(n=5$, one of the patients had a pathogenic PHEX mutation, the remaining four patients who refused genetic testing was diagnosed according to the biochemical characteristics), ${ }^{4}$ Fanconi syndrome with the features of renal tubular acidosis and vitamin D deficiency $(\mathrm{n}=4)$, and hereditary vitamin D abnormality $(n=12)$. Patients with underlying bone metabolism imbalance stemming from chronic disease and/or tumors were excluded. ${ }^{7}$ Additionally, patients with hyperparathyroidism or receiving medication known to affect bone metabolism were also excluded.

All measurements were collected using standard laboratory methods. During an initial clinical visit, age, sex, height, weight, current medications, clinical diagnosis, serum P levels, serum Ca levels, serum alkaline phosphatase (ALP) and parathyroid hormone (PTH) levels were measured. X-ray was used to confirm any reported bone deformities. Blood samples (second morning void) were obtained between 08:00 and 09:00 after an overnight fast.

\section{Bone Area, Mineral Content and Density} Bone area $\left(\mathrm{BA}, \mathrm{cm}^{2}\right)$, bone mineral content $(\mathrm{BMC}, \mathrm{g})$ and BMD of participants were measured using dual-energy X-ray absorptiometry (DXA) (Prodigy, GE Lunar, Madison, WI, USA). BMD of the projected bone area 
was calculated as $\mathrm{BMC} / \mathrm{BA}\left(\mathrm{g} \mathrm{cm}^{-2}\right)$. Skeletal sites included the lumbar spine (L1-L4), the femoral neck and total left hip were also examined. Depending on body thickness and the area scanned, scanning times ranged from 3 to $20 \mathrm{~min}$. Total radiation dose for each participant was less than $1 \mu \mathrm{Sv}$.

\section{Follow-Up}

Each patient was given continuous calcitriol $(0.25 \mu \mathrm{g}$, qd), calcium $\left(\mathrm{CaCO}_{3}, 0.3 \mathrm{~g}\right.$, bid) and a self-blended neutral phosphate solution $\left(0.8 \mathrm{M} \mathrm{Na}_{2} \mathrm{HPO}_{4}, 0.6 \mathrm{M} \mathrm{KH}_{2} \mathrm{PO}_{4}\right.$, $\mathrm{pH}=7.2)$ treatment. The protocol for the formulation of the phosphorus solutions was described as follows: First of all, the patients need to weigh 253 grams of $\mathrm{Na}_{2} \mathrm{HPO}_{4}$ and 126 grams of $\mathrm{KH}_{2} \mathrm{PO}_{4}$ respectively, and then mix them. The next step is to adjust the PH to 7.2 after adding $1000 \mathrm{~mL}$ drinking water to the mixture. Most importantly, we suggest the participants to administer the phosphate solution from 3 to $4 \mathrm{~mL}$ on, and take it every 5 hours. However, the dose will be adjusted according to the participants' serum phosphorus level, and usually the administration is to take the phosphate solution $6 \mathrm{~mL}$ each time and four times a day.

Eleven patients with $\mathrm{HO}$ were recalled for clinical assessment and divided into the following 3 groups according to their actual administration of phosphorus solution approximately three years after their initial visits:

- Patients in group A $(\mathrm{n}=3)$ received $6 \mathrm{~mL}$ phosphate solution doses at a frequency dependent upon measured serum phosphorus levels. If serum phosphorus was below $0.7 \mathrm{mmol} \mathrm{L} \mathrm{L}^{-1}$, phosphate solution was administered four times a day; daily dose count was reduced by one for each $0.1 \mathrm{mmol} \mathrm{L}^{-1}$ increase in serum phosphorus.

- Patients in group B $(n=5)$ received phosphate solution doses only every three to four days.

- Patients in group $C(n=3)$ received no phosphate solution. Patients in this group chose not to take the phosphate solution due to difficulties in preparing the solution in low-resource environments.

$\mathrm{BA}, \mathrm{BMC}$, and BMD of each follow-up participant were measured by the same licensed doctor from the department of endocrinology using the same DXA scanner as during the initial visit. Each participant's fracture history and medication history were recorded.

\section{Statistical Analysis}

Data analyses were carried out using SPSS version 17.0 for Windows (IBM Corp., Armonk, NY, USA). Student's $t$-test was used to compare BA, BMC and BMD levels in the different body sites between the case and control groups. Changes between the initial-visit and follow-up serum $\mathrm{P}, \mathrm{Ca}$, and ALP concentrations, as well as BMD in the different body sites were analyzed using paired sample $t$-test. All tests were two-tailed, and $\mathrm{P}<0.05$ was considered significant.

\section{Results \\ Participants}

Twenty-one patients (13 males and 8 females) in case group were diagnosed with HO. The duration of illness averaged $24 \pm 13$ (mean \pm standard deviation) years. All HO patients had some bone pain history. Sixteen of them had some bone deformity: vertebral deformities (62.5\%), lower limb deformities (25.0\%), and rib-sternal deformities $(12.5 \%)$. Six patients had a history of bone fracture: 3 with vertebra fractures, 2 with femur fractures, and 1 with a rib-sternal fracture.

Table 1 shows the demographic and biochemical data for all participants by gender. Ages of $21 \mathrm{HO}$ patients ranged from 13 to 50 years with an average of $37 \pm 10$ years. No significant difference in serum $\mathrm{P}, \mathrm{Ca}$ and $\mathrm{PTH}$ was observed between sexes, but serum ALP of females was significantly higher than that of males. Average baseline serum P level of case group $\left(0.59 \pm 0.18 \mathrm{mmol} \mathrm{L}^{-1}\right)$ was about $25 \%$ lower than that of control group. The case group average baseline serum ALP and PTH levels were $238.95 \pm 74.08 \mathrm{u} \mathrm{L}^{-1}$ and $11.04 \pm 5.71 \mathrm{pmol} \mathrm{L}^{-1}$, respectively, which were significantly higher than those of control group.

\section{Bone Area, Mineral Content, and Density}

Table 2 suggests that there were no significant differences in BA between the $\mathrm{HO}$ and control groups $(P>0.05)$, while the BMC measurements of L1-L4 and femur neck region in patients with $\mathrm{HO}$ were significantly lower than those of control group $(P<0.05)$. BMD in the L1-L4 regions was $10.4 \%$ $(P<0.01)$ lower in the HO group than in the control group. Meanwhile, the average BMD of the femur neck was $22.4 \%$ decreased in case group than that in control group. The average BMD of the total hip was $21.2 \%$ lower in HO group than in the control group. Our results indicated that BMD of the femur neck showed the greatest difference $(P<0.05)$ between the $\mathrm{HO}$ and control groups. 
Table I Distribution of Demographic and Biochemical Data of Patients According to Sex

\begin{tabular}{|l|c|c|c|c|c|c|c|}
\hline Sex & $\mathbf{n}$ & Height $\mathbf{( c m )}$ & Weight $\mathbf{( k g )}$ & $\begin{array}{c}\text { Serum P } \\
\left(\mathbf{m m o l} \mathbf{L}^{-1}\right)\end{array}$ & $\begin{array}{c}\text { Serum Ca } \\
\left(\mathbf{m m o l} \mathbf{L}^{-1}\right)\end{array}$ & $\begin{array}{c}\text { Serum ALP } \\
\left(\mathbf{u} \mathbf{L}^{-1}\right)\end{array}$ & $\begin{array}{c}\text { PTH } \\
\left(\mathbf{p m o l} \mathbf{L}^{-1}\right)\end{array}$ \\
\hline Patients & & & & & & \\
Males & 13 & $161.1 \pm 8.7$ & $60.4 \pm 8.6$ & $0.59 \pm 0.18$ & $2.02 \pm 0.32$ & $235.83 \pm 83.56$ & $9.94 \pm 5.82$ \\
Females & 8 & $140.8 \pm 18.2$ & $52.7 \pm 22.0$ & $0.60 \pm 0.22$ & $2.10 \pm 0.29$ & $244.43 \pm 65.31$ & $14.68 \pm 4.13$ \\
Total & 21 & $153.0 \pm 16.4$ & $57.3 \pm 154$ & $0.59 \pm 0.18$ & $2.05 \pm 0.30$ & $238.95 \pm 74.08^{\mathrm{b}}$ & $11.04 \pm 5.71^{\mathrm{b}}$ \\
\hline Controls & & & & & & \\
Males & 65 & $163.8 \pm 4.5$ & $62.7 \pm 6.5$ & $1.16 \pm 0.11$ & $2.18 \pm 0.09$ & $75.99 \pm 19.60$ & $3.88 \pm 1.38$ \\
Females & 40 & $152.5 \pm 5.6$ & $52.1 \pm 9.0$ & $1.12 \pm 0.13$ & $2.22 \pm 0.11$ & $72.31 \pm 26.98$ & $3.86 \pm 1.26$ \\
Total & 105 & $159.5 \pm 7.4$ & $58.7 \pm 9.1$ & $1.14 \pm 0.12$ & $2.20 \pm 0.10$ & $74.08 \pm 23.34$ & $3.87 \pm 1.30$ \\
\hline
\end{tabular}

Note: ${ }^{b} P<0.01$.

Abbreviations: P, phosphorus; Ca, calcium; ALP, alkaline phosphatase; PTH, parathyroid hormone.

\section{Serum Biomarkers}

Correlations between serum biochemical indices $(\mathrm{P}, \mathrm{Ca}$, and ALP) and BMD at various sites were presented in Table 3 (patient group, $\mathrm{n}=21$ ). Serum $\mathrm{P}$ level was positively correlated with baseline femur neck BMD $(\mathrm{r}=0.575, P<0.05)$, but no significant correlations were observed between serum $\mathrm{P}$ and BMD at the other tested sites.

Correlations between the first-visit serum Ca and ALP levels and BMD of the different sites were not significant, suggesting that first-visit serum Ca and ALP levels may not directly influence BMD.

\section{Follow-Up}

Eleven of $21 \mathrm{HO}$ patients returned for follow-up. Figure 1 shows the measured BMD changes during the treatment

Table 2 Distribution of BA, BMC and BMD Data in Different Sites for Patient and Control Groups

\begin{tabular}{|l|c|c|c|}
\hline Measurements & $\begin{array}{c}\text { Patient } \\
(\mathbf{n}=\mathbf{2} \text { ) })\end{array}$ & $\begin{array}{c}\text { Controls } \\
(\mathbf{n}=105)\end{array}$ & $P$ value \\
\hline LI-L4 & & & \\
BA $\left(\mathrm{cm}^{2}\right)$ & $57.22 \pm 5.29$ & $58.15 \pm 7.46$ & 0.200 \\
BMC (g) & $47.07 \pm 15.48$ & $52.86 \pm 11.85$ & $0.048^{\mathrm{a}}$ \\
BMD $\left(\mathrm{g} \mathrm{cm}^{-2}\right)$ & $0.807 \pm 0.256$ & $0.901 \pm 0.126$ & $0.000^{\mathrm{b}}$ \\
\hline Femur Neck & & & \\
BA $\left(\mathrm{cm}^{2}\right)$ & $5.07 \pm 0.58$ & $5.04 \pm 0.46$ & 0.459 \\
BMC $(\mathrm{g})$ & $3.07 \pm 1.04$ & $3.84 \pm 0.73$ & $0.041^{\mathrm{a}}$ \\
BMD $\left(\mathrm{g} \mathrm{cm}^{-2}\right)$ & $0.589 \pm 0.173$ & $0.759 \pm 0.117$ & $0.031^{\mathrm{a}}$ \\
\hline Total Hip & & & \\
BA $\left(\mathrm{cm}^{2}\right)$ & $37.78 \pm 5.55$ & $35.79 \pm 5.17$ & 0.774 \\
BMC $(\mathrm{g})$ & $25.57 \pm 8.69$ & $31.87 \pm 7.59$ & 0.796 \\
BMD $\left(\mathrm{g} \mathrm{cm}^{-2}\right)$ & $0.673 \pm 0.184$ & $0.854 \pm 0.129$ & $0.014^{\mathrm{a}}$ \\
\hline
\end{tabular}

Notes: ${ }^{a} P<0.05 ;{ }^{b} p<0.01$.

Abbreviations: LI-L4, lumbar spine; BA, Bone area; BMC, bone mineral content; BMD, bone mineral density. period at the different anatomical sites. Compared with the initial pre-treatment measurement, the BMD of patients who were prescribed phosphate supplementation was noted to increase after treatment. Patients in group A showed total BMD increased between $37.9 \%$ and $52.3 \%$. In particular, an increase of $43.2-79.3 \%$ was noted for the femoral neck. One participant in group B $(n=5)$ had previously received phosphate therapy but discontinued the treatment upon relief of pain, and phosphate supplementation was accepted again when the bone pain subsequently intensified. The rest of 4 patients in group B were initially treated consistently with a neutral phosphate solution, followed by a period of intermittent supplementation. The BMD of these patients was noted to increase between $4.9 \%$ and $33.5 \%$ in the total hip, and

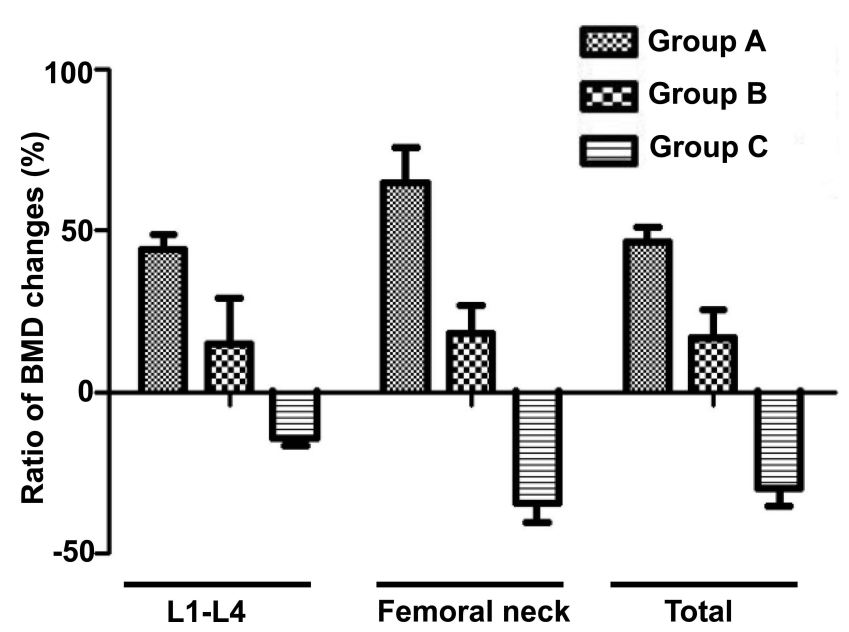

Figure I Observed BMD changes of $\mathrm{HO}$ patients after different treatments. BMD of $\mathrm{LI}-\mathrm{L} 4$, the femoral neck and the total hip significantly increased $(P<0.0 \mathrm{I})$ after continuous treatment with a neutral phosphate solution (group A). There was no significant improvement in the BMD after intermittent treatment with a neutral phosphate solution (group B, $P>0.05$ ). Patients not treated with a neutral phosphate solution showed decreased BMD (group $C, P<0.05$ ). 
Table 3 Correlation Between Serum P, Ca and ALP Levels and BMD at Different Anatomical Sites

\begin{tabular}{|l|c|c|c|c|c|c|}
\hline & \multicolumn{2}{|c|}{ LI-L4 } & \multicolumn{2}{c|}{ Femur Neck } & \multicolumn{2}{c|}{ Total } \\
\cline { 2 - 7 } & $\boldsymbol{r}$ & $\boldsymbol{P}$ value & $\boldsymbol{r}$ & $\boldsymbol{P}$ value & $\boldsymbol{r}$ & $\boldsymbol{P}$ value \\
\hline Serum P & 0.135 & 0.605 & 0.575 & $0.044^{\mathrm{a}}$ & 0.109 & 0.699 \\
Serum Ca & -0.207 & 0.425 & -0.392 & 0.120 & -0.415 & 0.124 \\
Serum ALP & 0.478 & 0.137 & 0.665 & 0.175 & 0.469 & 0.203 \\
\hline
\end{tabular}

Note: ${ }^{\mathrm{a} P}<0.05$.

Abbreviations: P, phosphorus; Ca, calcium; ALP, alkaline phosphatase; BMD, bone mineral density; LI-L4, lumbar spine.

between $17.7 \%$ and $33.4 \%$ in the femoral neck. However, $\mathrm{BMD}$ measurements of group $\mathrm{C}$ were noted to decrease in the femoral neck $(-41.7 \%$ to $-22.5 \%)$. During treatment, one participant in group $\mathrm{C}$ had fractures of the right ribs 8 10 and the left ribs 7-9 caused by a turbulent bus ride. No other follow-up patients in any of the three groups had fractures during the treatment period (Figure 1).

\section{Discussion}

The aim of our study is to investigate the changes in BMD in HO patients before and after treatment. Many investigators have shown that BMD is markedly reduced in osteomalacic bone..$^{7-13}$ It has been reported that up to $70 \%$ of osteomalacia patients had BMD with osteoporosis in their lumbar spine, and up to $60 \%$ of osteomalacia patients had BMD with osteoporosis in their femoral neck by a study from Iran. ${ }^{9}$ The results from this study also showed that the BMD of HO patients is markedly reduced before treatment and increased after conventional treatment with oral phosphate and calcitriol. This indicated that BMD is decreased in most osteomalacia and it may help in assessing the bone status before and after treatment.

Although bone biopsy is the most reliable test for bone mineral deficits monitoring in osteomalacia patients, its routine clinical application is limited due to the invasive nature. Compared with bone biopsy, BMD measured by DXA is a non-invasive method with minimal radiation exposure. It is reported that BMD of the lumbar spine and femur significantly correlated with quantitative histological measurement in patients with a variety of metabolic bone diseases including osteomalacia. ${ }^{14}$ In our study, BMD in the L1-L4, total hip and femur neck regions in HO patients were significantly lower than that in healthy individuals before treatment with the BMD in femur neck decreased the most obvious. These results are equal to the previous research in which BMD measurements both at the lumbar spine and the femoral neck are in the osteoporotic range in a Caucasian patient with HO. ${ }^{11}$ After treatment with neutral phosphate solution concurrent with calcitriol and calcium, both the BMD in L1-L4 and total hip showed an improvement in which the femoral neck BMD increased the most (43.2-79.3\%). These results indicated that $\mathrm{BMD}$ might contribute to the retrospective confirmation of the bone mineral deficits, especially femoral neck BMD appeared to be better markers to monitor the therapeutic efficacy of HO. Thus, BMD could be used for clinicians to determine when to reduce phosphate supplementation so as to avoid overtreatment and maintain serum phosphate levels close to the lower limit of normal.

Treatment is recommended in hypophosphatemia adults with symptoms including osteomalacia. However, there are limited clinical studies demonstrated the efficacy of conventional therapy with oral phosphate and calcitriol in adults with HO. ${ }^{5,11}$ Therefore, our study performed conventional therapy on patients with $\mathrm{HO}$ and evaluated its efficiency. Because commercialized oral phosphate supplements are unavailable in developing areas such as in rural China, a self-blended phosphate solution according to the recommended daily elemental phosphorus ( 750 to $1000 \mathrm{mg} / \mathrm{d}$ in 3 to 4 divided dosages) ${ }^{6}$ for $\mathrm{HO}$ was used for the treatment so that patients themselves prepared the oral phosphate solution at home. Femur neck BMD was found to significantly positive correlate with serum P levels before treatment. Reduced serum phosphorous leads to activation of PTH and 1, 25dihydroxyvitamin $\mathrm{D}\left(1,25(\mathrm{OH})_{2} \mathrm{D}\right)$, and consequently causes a reduction of serum $\mathrm{Ca}^{15}$ Reduction of serum $\mathrm{Ca}$ further increases the synthesis and secretion of PTH, which results in receptor activation for nuclear factor- $\kappa \mathrm{B}$ ligand (RANKL) by osteoblast cells, thus enhancing osteoclast formation and bone resorption. ${ }^{16}$ Increasing serum P levels, then, is an important component of treatment plans to increase and maintain BMD in patients with HO. Therefore, the self-blended neutral phosphate 
solution used for the treatment was adjusted according to measured serum P levels. After treatment, we found that patients who received calcitriol and calcium without neutral phosphate solution showed decreases in BMD, and in one case, multiple fragility fractures. In group B, since the patients were only intermittently administrated neutral phosphate solution, there was no significant improvement in the BMD after treatment. This may be related to the fact that the serum phosphorus levels of patients who take neutral phosphate solution intermittently cannot reach the normal level. Similarly, the study of Bhambri et al discovered that within the first few months of treatment, there was a huge improvement in clinical syndrome and biochemical markers in osteomalacia patients, but the bone mineral defects take much longer to repair and the ultimate extent of recovery at different skeletal sites varies. ${ }^{7}$ Therefore, it is of vital importance to continue treatment until bone mineral defects are satisfactorily improved. While consistent supplementation with neutral phosphate solution, calcitriol and calcium could increase the BMD of $\mathrm{HO}$ patients, especially in the femoral neck, and the effect appears to be partially maintained once treatment is discontinued. This is consistent with the study by Sullivan et al and Harrell et al, who showed that calcitriol and phosphorus supplements could reverse the extent of mineralization defect detected by bone biopsy in HO. ${ }^{5,17}$ Meanwhile, none of the previously reported side-effects such as hyperparathyroidism, nephrocalcinosis, or other diseases ${ }^{18,19}$ were observed during therapy. These results indicated that conventional therapy combined calcitriol and phosphate could improve bone mineralization in both the lumbar spine and hip bone in $\mathrm{HO}$.

This study had a few notable limitations. The duration of illness and time for follow-up were uncontrolled and only 11 of $21 \mathrm{HO}$ patients returned for follow-up. Small sample sizes, especially in the HO subgroups limit the statistical power of our results, and preclude adjustment for sex, age and menopause status. Further research with larger sample size is needed to better understand the efficacy of phosphate supplementation and optimize treatment regimens for $\mathrm{HO}$ patients of various demographics.

\section{Conclusion}

In conclusion, the results from this study suggest that BMD measured by DXA may be a good noninvasive marker to assess the severity of bone mineral defects before and after treatment. Furthermore, evaluating changes in BMD with combined intermittent and continuous self-blended neutral phosphate solution and calcitriol therapy in $\mathrm{HO}$ patients show that continuous conventional medical therapy is beneficial in the treatment of mineralization defects in patients with $\mathrm{HO}$ in China, as proved by the improvement of BMD in the lumbar spine, hip and femoral neck. Therefore, we suggest that BMD changes should be monitored in patients with $\mathrm{HO}$ at regular intervals to evaluate the therapeutic effect and treatment should be continued until BMD is satisfactorily improved.

\section{Abbreviations}

$\mathrm{HO}$, hypophosphatemic osteomalacia; BMD, bone mineral density; P, phosphorus; Ca, calcium; DXA, dual-energy X-ray absorptiometry; ALP, alkaline phosphatase; PTH, parathyroid hormone; BA, Bone area; $\mathrm{BMC}$, bone mineral content; L1-L4, lumbar spine; 1,25(OH)2D, 1,25-dihydroxyvitamin D; RANKL, receptor activation for nuclear factor- $\kappa \mathrm{B}$ ligand.

\section{Data Sharing Statement}

The datasets generated and/or analysed during the current study are available in the ResMan repository, http://www. $\underline{\text { medresman.org/pub/cn/proj/projectshow.aspx?proj=2108. }}$.

\section{Ethics Approval and Informed Consent}

The study was approved by the Ethics Committee of The Second Xiangya Hospital of Central South University. Written informed consent was obtained from the subjects before all measurements.

\section{Author Contributions}

Hou-De Zhou: Conceptualization, Supervision and Funding acquisition. Yue Guo and Ying-Hui Zhou: Methodology and Formal analysis. Yue Guo, Ying-Hui Zhou, Xian-Ping Wu, Chen-Yi Tang, Min Wang, Zhao-Hui Mo and John A Shepherd: Investigation. Yue Guo, Ying-Hui Zhou, Bennett $\mathrm{K} \mathrm{Ng}$ and Bo Fan: Interpretation. All authors participated in "Writing and Editing" of the manuscript and agreed to submit the manuscript to "International Journal of General Medicine". All authors reviewed and agreed on all versions of the manuscript before submission, during revision, the final version accepted for publication, and any significant changes introduced at the proofing stage. All authors agree to take responsibility and be accountable for the contents of the manuscript. 


\section{Funding}

This work was supported by the National Natural Scientific Foundation of China [grant numbers: 81770880, 81800788, 81970762], the Science \& Technology Department of Hunan Province [grant numbers: 2020SK2080, 2015JC3012 and 2018SK52511] and Changsha City [grant numbers: k1906019, kq1901118], and the Open Sharing Fund for the Large-scale Instruments and Equipment of Central South University.

\section{Disclosure}

The authors report no conflicts of interest in this work.

\section{References}

1. Kinoshita Y, Fukumoto S. X-linked hypophosphatemia and FGF23-related hypophosphatemic diseases: prospect for new treatment. Endocr Rev. 2018;39(3):274-291. doi:10.1210/er.201700220

2. Bockenhauer D, Bokenkamp A, Van't Hoff W, et al. Renal phenotype in Lowe syndrome: a selective proximal tubular dysfunction. Clin $J$ Am Soc Nephrol. 2008;3(5):1430-1436. doi:10.2215/CJN.00520108

3. Bergwitz C, Roslin NM, Tieder M, et al. SLC34A3 mutations in patients with hereditary hypophosphatemic rickets with hypercalciuria predict a key role for the sodium-phosphate cotransporter NaPi-IIc in maintaining phosphate homeostasis. Am J Hum Genet. 2006;78 (2):179-192. doi:10.1086/499409

4. Shanbhogue VV, Hansen S, Jorgensen NR, Beck-Nielsen SS. Impact of conventional medical therapy on bone mineral density and bone turnover in adult patients with X-linked hypophosphatemia: a 6-year prospective cohort study. Calcif Tissue Int. 2018;102(3):321-328. doi:10.1007/s00223-017-0363-3

5. Sullivan W, Carpenter T, Glorieux F, Travers R, Insogna K. A prospective trial of phosphate and 1,25-dihydroxyvitamin D3 therapy in symptomatic adults with X-linked hypophosphatemic rickets. J Clin Endocrinol Metab. 1992;75(3):879-885.

6. Carpenter TO, Imel EA, Holm IA, Jan de Beur SM, Insogna KL. A clinician's guide to X-linked hypophosphatemia. J Bone Miner Res. 2011;26(7):1381-1388. doi:10.1002/jbmr.340
7. Bhambri R, Naik V, Malhotra N, et al. Changes in bone mineral density following treatment of osteomalacia. J Clin Densitom. 2006;9 (1):120-127. doi:10.1016/j.jocd.2005.11.001

8. Jeong HJ, Lee JM, Lee TH, et al. Two cases of hypophosphatemic osteomalacia after long-term low dose adefovir therapy in chronic hepatitis B and literature review. $J$ Bone Metab. 2014;21(1):76-83. doi:10.11005/jbm.2014.21.1.76

9. Saghafi M, Azarian A, Hashemzadeh K, Sahebari M, Rezaieyazdi Z. Bone densitometry in patients with osteomalacia: is it valuable? Clin Cases Miner Bone Metab. 2013;10(3):180-182.

10. Arthur S, Chopra A. Familial hypophosphatemia: an unusual presentation with low back ache, heel pain, and a limp in a young man, and literature review. Clin Rheumatol. 2011;30(4):585-589. doi:10.1007/ s10067-010-1607-z

11. Negri AL, Bogado CE, Zanchetta JR. Bone densitometry in a patient with hypophosphatemic osteomalacia. J Bone Miner Metab. 2004;22 (5):514-517. doi:10.1007/s00774-004-0516-0

12. El-Desouki MI, Othman SM, Fouda MA. Bone mineral density and bone scintigraphy in adult Saudi female patients with osteomalacia. Saudi Med J. 2004;25(3):355-358.

13. El-Desouki M, Al-Jurayyan N. Bone mineral density and bone scintigraphy in children and adolescents with osteomalacia. Eur J Nucl Med. 1997;24(2):202-205. doi:10.1007/BF02439554

14. Cosman F, Schnitzer MB, McCann PD, Parisien MV, Dempster DW, Lindsay R. Relationships between quantitative histological measurements and noninvasive assessments of bone mass. Bone. 1992;13 (3):237-242. doi:10.1016/8756-3282(92)90203-9

15. Fukumoto S. The role of bone in phosphate metabolism. Mol Cell Endocrinol. 2009;310(1-2):63-70. doi:10.1016/j.mce.2008.08.031

16. Martin A, David V, Quarles LD. Regulation and function of the FGF23/klotho endocrine pathways. Physiol Rev. 2012;92 (1):131-155. doi:10.1152/physrev.00002.2011

17. Harrell RM, Lyles KW, Harrelson JM, Friedman NE, Drezner MK. Healing of bone disease in X-linked hypophosphatemic rickets/osteomalacia. Induction and maintenance with phosphorus and calcitriol. J Clin Invest. 1985;75(6):1858-1868. doi:10.1172/JCI111900

18. Imel EA, DiMeglio LA, Hui SL, Carpenter TO, Econs MJ. Treatment of X-linked hypophosphatemia with calcitriol and phosphate increases circulating fibroblast growth factor 23 concentrations. $J$ Clin Endocrinol Metab. 2010;95(4):1846-1850. doi:10.1210/ jc.2009-1671

19. Faul C, Amaral AP, Oskouei B, et al. FGF23 induces left ventricular hypertrophy. J Clin Invest. 2011;121(11):4393-4408. doi:10.1172/ JCI46122
International Journal of General Medicine

\section{Publish your work in this journal}

The International Journal of General Medicine is an international, peer-reviewed open-access journal that focuses on general and internal medicine, pathogenesis, epidemiology, diagnosis, monitoring and treatment protocols. The journal is characterized by the rapid reporting of reviews, original research and clinical studies across all disease areas. The manuscript management system is completely online and includes a very quick and fair peer-review system, which is all easy to use. Visit http://www.dovepress.com/ testimonials.php to read real quotes from published authors. 